


\section{LA BIOLOGÍA DEL DESARROLLO COMO MEDIO PARA ABORDAR EL CONOCIMIENTO DEL CUERPO HUMANO EN ESTUDIANTES DE OCTAVO GRADO DEL COLEGIO MANUELA BELTRÁN}

\section{Development Biology as a Means of Addressing the Knowledge of the Human Body in Eighth Grade Students of the Manuela Beltran School}

\section{A biologia do desenvolvimento como meio para abordar o conhecimento do corpo humano em alunos da oitava série do Colégio Manuela Beltran}

\section{Angie Daniela Vargas-Guzmán*}

Fecha de recepción: 23 de enero de 2020

Fecha de aceptación: 14 de junio de 2020

\section{Cómo citar este artículo:}

Vargas-Guzmán, A. D. (2020). La biología del desarrollo como medio para abordar el conocimiento del cuerpo humano en estudiantes de grado octavo del Colegio Manuela Beltrán. Bio-grafía, 13(25). 135-141. https://doi.org/10.17227/bio-grafia.vol.13.num25-12192

\section{Resumen}

En este artículo de investigación se muestra un proyecto que se realizó durante la práctica pedagógica para abordar el conocimiento del cuerpo humano a través de la biología del desarrollo. Se hace esta propuesta considerando la importancia que tiene para el docente de biología el elaborar material didáctico con el objetivo de vincular a los estudiantes en su proceso de aprendizaje y, de esta manera, permitir una enseñanza que involucre las problemáticas de la adolescencia (baja autoestima, bajo rendimiento académico, etc.) encontradas en el aula. En primer lugar, se hizo una contextualización en el aula para identificar algunas de las problemáticas de octavo grado, luego se hizo una búsqueda bibliográfica sobre la biología del desarrollo y, por último, se elaboró una unidad didáctica titulada "El mundo de la biología del desarrollo humano". En la unidad se abordaron los conceptos fundamentales del tema y se incluyó una sección de preguntas basadas en estudios de caso, datos curiosos y tips para el cuidado del cuerpo humano. La elaboración de la unidad didáctica se basó en lo que habría ocurrido en una posible aplicación, pues debido a la pandemia por la covid-19 no fue posible su implementación.

Palabras clave: biología del desarrollo; enseñanza de la biología; cuerpo humano; adolescencia

* Estudiante de la Licenciatura en Biología de la Universidad Pedagógica Nacional. Correo electrónico: advargasg@upn.edu.co 


\section{Abstract}

This paper shows a project that was carried out during the teaching practicum to address the knowledge of the human body through the biology of development. This proposal is made considering how important it is for the biology teacher to create teaching material to involve students in their own learning process. This will make possible a teaching which takes into consideration the problems of adolescence found in the classroom (i. e., low self-esteem, poor academic performance, etc.). To begin, it was necessary to do a contextualization in the classroom to identify some of the problems found in the eighth grade. Then, the author did a bibliographic search -about developmental biology. Finally, she developed a teaching unit called "El mundo de la biología del desarrollo humano", which addressed the core concepts of the topic. It contained a question section based on case studies, interesting facts and tips for caring the human body. The development of the teaching unit was based on what would have happened in a possible application, since the emergence of the covid-19 pandemic prevented its implementation.

Keywords: developmental biology; biology teaching; human body; adolescence

\section{Resumo}

Neste artigo de pesquisa mostra-se um projeto que foi realizado durante o estágio pedagógico para abordar o conhecimento do corpo humano por meio da biologia do desenvolvimento. Esta proposta é feita considerando a importância que tem para o professor de biologia produzir material didático de forma com o objetivo de associar os alunos em seu processo de aprendizagem, e dessa forma, possibilitar um ensino que envolva as problemáticas da adolescência ( baixa autoestima, baixo rendimento escolar, etc.) encontradas na sala aula. Em primeiro lugar, foi feita uma contextualização em sala de aula para identificar alguns dos problemas da oitava série, depois foi realizada uma pesquisa bibliográfica sobre biologia do desenvolvimento e, por fim, foi desenvolvida uma unidade didática intitulada " O mundo da biologia do desenvolvimento humano". Na unidade abordaram-se os conceitos fundamentais do tema incluindo um bloco de perguntas baseadas em estudos de caso, curiosidades e dicas para o cuidado do corpo humano. A produção da unidade didática teve como base o que teria acontecido em uma possível aplicação, pois devido a pandemia do COVID-19 sua implementação não foi possível.

Palavras-chave: biologia do desenvolvimento; ensino de biologia; corpo humano; adolescência 
El presente artículo muestra la importancia de llevar al aula la enseñanza de la biología del desarrollo por un método alterno al tradicional, entendido por Ríos y Urdaneta como aquel en el cual

el estudiante es un elemento cognitivo pasivo, que debe aprender cada lección enseñada al pie de la letra; es decir, es un receptor responsable del aprendizaje. En este sentido, el estudiante es un reproductor de los saberes transmitidos en la escuela, sin derecho a opinar e innovar respecto de los mismos. (2015, p. 918)

Por esta razón se diseñó una unidad didáctica en la que se presentan conceptos fundamentales para la comprensión de los procesos de desarrollo del cuerpo humano; se realizaron guías a partir de estudios de caso; se elaboraron consejos para cuidar el organismo de diferentes enfermedades; se emitieron datos curiosos acerca de células somáticas, células sexuales, la gestación y reflexiones frente a la sexualidad.

El primer aspecto que se aborda es el contexto nacional desde los Derechos Básicos de Aprendizaje (Ministerio de Educación Nacional, 2016); el segundo, a partir de una contextualización en la institución educativa Manuela Beltrán y, por último, se presenta una ruta metodológica cuyo objetivo es dar una posible solución a las problemáticas encontradas en el contexto para abordar la enseñanza de esta temática por medio de la elaboración de una unidad didáctica realizada en la plataforma virtual Canva denominada "El mundo de la biología del desarrollo humano". Se seleccionó esta plataforma porque es de acceso libre, no establece un límite o número de páginas para poder descargar el material realizado, se pueden agregar imágenes y videos para complementar el texto, presenta muchas opciones para elegir algún diseño, color o tipo de letra y es fácil de manejar. No obstante, a la hora de elaborar guías o alguna forma de evaluación virtual, esta plataforma no es tan dinámica e interactiva. Cabe destacar que se realizó de forma virtual debido a la situación actual que atraviesa la educación por la coyuntura de la covid-19.

Se elaboró este material porque los lineamientos del Ministerio de Educación Nacional para el área de Ciencias Naturales establecen que las temáticas que deben ser abordadas por el maestro en el aula con estudiantes de octavo grado en Biología son las relacionadas con procesos vitales: crecimiento, nutrición y reproducción, homeóstasis, metabolismo, sistema nervioso y sistema endocrino como sistemas integradores del organismo.

Pese a que esto puede ser un aporte para que el estudiante aprenda sobre sus procesos de desarrollo, abordar estas temáticas de forma tradicional hace que se tengan dificultades en el aula para comprenderlas y relacionarlas con el conocimiento de sí mismo. Cuando no se utilizan metodologías que vinculen los conceptos vistos en el aula con el contexto o situaciones que les ocurren a los estudiantes, estos tenderán a verlos como complejos y poco pertinentes en su vida cotidiana, y lo visto en el aula no se tendrá en cuenta a la hora de abordar problemáticas que son propias de la adolescencia, como la baja autoestima, el maltrato escolar, la inseguridad en las relaciones, los sentimientos de soledad, la inestabilidad emocional y la desconfianza hacia los seres humano. Esto puede provocar sufrimientos emocionales y sociales que pueden favorecer el aislamiento, la depresión e incluso el suicidio, un tema tabú en nuestra sociedad (Sánchez, 2015).

Además, la adolescencia "es una etapa extremadamente rica que acabará consolidando su seguridad o inseguridad en sí mismo y en los demás. El humor, las emociones y los sentimientos conforman un mundo emocional muy variable de unos adolescentes a otros" (Sánchez, 2015, p. 11).

En consideración de las anteriores problemáticas, es necesario hacer propuestas pedagógicas y didácticas para una enseñanza de la biología del desarrollo que se ajuste al contexto en el que se va a abordar la temática y que, de esta manera, se puedan aportar conocimientos y reflexiones en torno a la complejidad de su organismo para generar una relación más sana, grata y agradable consigo mismo y en el aula.

La unidad didáctica puede aportar a esto, pues, como afirman Cáceres Péfaur et al.:

es una herramienta de planificación que permite considerar de manera integral todos los elementos que están implícitos en el proceso educativo, por lo que se convierte en una guía para el docente durante todas las fases o momentos del proceso educativo. A través de la unidad didáctica se planifica el trabajo cotidiano en el aula, tomando en cuenta todos los elementos del proceso de enseñanza-aprendizaje de manera coherente, facilitando la globalización de los conocimientos, así como la contextualización de los mismos a partir de la realidad que rodea al estudiante. (2016, p. 250)

Además, Cárdenas (2017) considera la importancia de que los maestros realicen una planeación acerca de la temática que se va abordar en el aula y cómo la unidad didáctica contribuye a esta organización y permite la participación de los estudiantes en su proceso de aprendizaje. Esto evidencia el potencial de este tipo de material, pues puede ser bien recibido en el aula. 
Por último, este proyecto se realiza desde la orientación del grupo de investigación Conocimiento Profesional del Profesor de Ciencias de la Universidad Pedagógica Nacional, cuyo objetivo es aportar a la producción de conocimiento didáctico de contenidos disciplinares específicos de las ciencias naturales y, en particular, de la biología, la física y la química.

\section{Metodología}

El enfoque de este proyecto fue cualitativo, pues Cerda (1993) lo caracteriza por los siguientes aspectos:

- La interpretación que se les da a las cosas y fenómenos no puede ser captada o expresada plenamente por la estadística o las matemáticas.

- Utiliza múltiples fuentes, métodos e investigadores para estudiar un solo problema o tema, los cuales convergen en torno a un punto central del estudio (principio de triangulación y convergencia).

- Centra el análisis en la descripción de los fenómenos y cosas observadas.

Para esto, se tuvo como corriente pedagógica el cognitivismo. De acuerdo con Gómez y Polanía:

plantea que la educación debe buscar que cada individuo acceda progresiva y secuencialmente a una etapa superior de su desarrollo intelectual de acuerdo con las necesidades y condiciones particulares de cada uno, lo cual a su vez se constituye en su meta educativa. [...] uno de los aportes más valiosos del modelo cognitivo es que destaca el carácter activo del sujeto en sus propios procesos de conocimiento y de desarrollo cognitivo, razón por la cual el maestro asume el rol de acompañante y facilitador. Debe generar en el estudiante situaciones de desequilibrio cognitivo, de cuestionamiento y revalidación de los propios conocimientos de manera tal, que el estudiante se vea obligado a explorar nuevas formas de resolver las situaciones problémicas, asimilar nuevos conocimientos con significados propios, construir y apropiar nuevos conceptos que, una vez estabilizados en un proceso de acomodación, se vean nuevamente cuestionados, puestos en desequilibrio, para que el estudiante inicie nuevos ciclos de construcción. (2008, pp. 63-64)

Respecto al modelo didáctico, se empleó el alternativo, pues, para Mayorga y Madrid:

la metodología didáctica se concibe como un proceso de "investigación escolar", es decir, no espontáneo, desarrollado por parte del alumno/a con la ayuda del profesor/a, lo que se considera como el mecanismo más adecuado para favorecer la "construcción" del conocimiento escolar propuesto; así, a partir del planteamiento de "problemas" (de conocimiento escolar) se desarrolla una secuencia de actividades dirigida al tratamiento de los mismos, lo que, a su vez, propicia la construcción del conocimiento manejado en relación con dichos problemas. (2010, p. 97)

Frente a la problemática central encontrada en el contexto educativo del Colegio Manuela Beltrán (enseñanza con un método tradicional y memorístico), la ruta metodológica propuesta tuvo en consideración la situación actual a la que se enfrenta la educación debido a la pandemia de la covid-19. Se elaboró una unidad didáctica en la plataforma virtual Canva, con el ánimo de contribuir al conocimiento del cuerpo desde la biología del desarrollo.

La elaboración de esta unidad didáctica se dio en las siguientes etapas:

\section{Contextualización (institución educativa y población estudiantil)}

El Colegio Manuela Beltrán opera en calendario A, es mixto y cuenta con dos sedes. El proyecto de práctica se realizará en la sede A, que ofrece dos jornadas (única y nocturna). El horario de la jornada única va de 6:30 a. m. a 2:30 p. m.

En el PEı de la institución, su misión se plantea así:

Somos una institución educativa distrital de carácter técnico comercial con formación humana integral e incluyentey de calidad para niños, niñas, jóvenes, adultos sordos y oyentes en los niveles de preescolar, básica y media, dirigida a desarrollar habilidades y saberes para la vida que promuevan una actitud emprendedora, con responsabilidad social, articulada con el SENA y otras instituciones. $(2018$, p. 8$)$

Para abordar la temática de biología del desarrollo humano, se observó que en esta institución educativa se veían conceptos relacionados con esta en grado octavo. Se seleccionó el curso 803 para realizar la práctica pedagógica debido a su horario. Este grupo lo conforman 32 estudiantes, y en la clase de biología se pone énfasis solo en memorizar y preguntar conceptos, sin ningún tipo de relación con las situaciones que se les puedan presentar a los estudiantes. También se evidencian problemáticas como la falta de interés y atención en la clase de biología, bajo rendimiento académico, poca participación, uso de objetos que pueden distraer a los estudiantes (celulares, esmaltes y planchas para el cabello), además de conflictos entre los compañeros. Estos conflictos se identificaron durante el periodo 2019-2. La información se tuvo por 
medio de diálogo con el docente de ciencias, la planilla de notas de la asignatura de biología y lo observado en el aula durante este periodo académico, tiempo en el cual asistimos dos veces por semana a la clase de biología hasta la culminación del año escolar. Como se mencionó, se decidió usar la unidad didáctica, en la que se incluyen documentales, situaciones problema, datos curiosos y personajes de ficción que buscan explicar los conceptos y, además, relacionarlos con el contexto de la adolescencia, pues fue con esta población que se realizó la intervención en el aula.

\section{Propuesta del material de acuerdo con las necesidades del contexto}

Consulta bibliográfica y selección de información de los siguientes libros:

Curtis, H. y Schnek, A. (2015). Biología. Editorial Médica Panamericana.

Sadler, T. W. y Langman, J. (2007). Langman: Embriología médica con orientación clínica. Editorial Médica Panamericana.

Sadler, T.W. y Langman J. (2007) Embriología médica/ Medical Embryology: Con orientación clínica. Editorial Médica Panamericana.
Tortora, G. J. y Derrickson, B. (2013). Principios de anatomía y fisiología. Editorial Médica Panamericana.

Rodríguez, M., Fernández, M. T. y Rivadeneira, F. (s. f.). Agentes teratogénicos y teratogenicidad, (10). Colección Derecho a Vivir en Desventaja.

\section{Elaboración de guías}

Estas guías buscaron identificar por medio de estudios de caso la comprensión de cada uno de los temas que se aborda en la unidad didáctica. Esto se hizo debido a que en la contextualización se evidenció que los estudiantes mostraban más interés frente a determinados temas por medio de ejemplos, documentales, imágenes, entre otros, que memorizando los conceptos únicamente. Cabe destacar que las guías propuestas son fundamentales para evaluar las temáticas que se presentan en cada una de secciones de la unidad didáctica ${ }^{1}$.

1 La elaboración de la unidad didáctica está disponible en https://drive.google.com/file/d/1sLIOuxjzA6rgV_XsAlfZdBm_ f4_PSebh/view?usp=sharing.

\section{Cuadro 1. Estructura de la unidad didáctica}

\begin{tabular}{|c|c|}
\hline Sección & Objetivo(s) \\
\hline $\begin{array}{l}\text { I. Me conozco y conozco al otro. } \\
\text { Estructura interna y externa de los sistemas } \\
\text { reproductivos masculinos y femeninos }\end{array}$ & $\begin{array}{l}\text { 1. Conocer la estructura y el funcionamiento de los sistemas reproductores masculino y femenino. } \\
\text { 2. Dar consejos para el cuidado de los sistemas reproductores femenino y masculino. } \\
\text { 3. Mostrar datos curiosos del sistema reproductor masculino. }\end{array}$ \\
\hline II. Conozco los procesos de división celular & $\begin{array}{l}\text { 1. Conocer cómo se producen los procesos de división celular en células somáticas y sexuales. } \\
\text { 2. Dar consejos para el cuidado y buen funcionamiento celular (prevención de hábitos, alimentos } \\
\text { y sustancias que puedan provocar cáncer). }\end{array}$ \\
\hline $\begin{array}{l}\text { III. Conozco los procesos del desarrollo } \\
\text { embrionario }\end{array}$ & $\begin{array}{l}\text { 1. Conocer las etapas y procesos del desarrollo embrionario. } \\
\text { 2. Establecer procesos de reflexión frente a la sexualidad y la responsabilidad que trae el embarazo. }\end{array}$ \\
\hline $\begin{array}{l}\text { Iv. Pero, ¿qué falló? } \\
\text { Un recorrido por las anormalidades } \\
\text { cromosómicas }\end{array}$ & $\begin{array}{l}\text { 1. Conocer las anomalías que se pueden presentar en el desarrollo embrionario y cómo } \\
\text { identificarlas. }\end{array}$ \\
\hline $\begin{array}{l}\text { v. ¡Culpemos al ambiente! } \\
\text { ¿Cómo se explican los contextos acerca de } \\
\text { las sustancias teratógenas? }\end{array}$ & $\begin{array}{l}\text { 1. Identificar los factores externos que pueden provocar anomalías congénitas. } \\
\text { 2. Conocer el contexto histórico que produjo el descubrimiento de estas sustancias. }\end{array}$ \\
\hline $\begin{array}{l}\text { vi. Ley de acción-reacción } \\
\text { Sobre los métodos anticonceptivos y la } \\
\text { responsabilidad social }\end{array}$ & 1. Reconocer la responsabilidad y la complejidad de traer vida. \\
\hline $\begin{array}{l}\text { VII. ¿A qué se deben los cambios que se } \\
\text { presentan en la adolescencia? } \\
\text { El sistema endocrino }\end{array}$ & $\begin{array}{l}\text { 1. Comprender cómo influye el sistema endocrino en la adolescencia a partir de guías de } \\
\text { indagación de ideas previas, estudios de caso y un refuerzo acerca del funcionamiento del } \\
\text { sistema endocrino. }\end{array}$ \\
\hline
\end{tabular}

Fuente: elaboración propia. 


\section{Resultados}

Se elaboró una unidad didáctica para abordar la temática de la biología del desarrollo. Tanto docentes como estudiantes pueden acceder a ella por medio del siguiente enlace: https://drive.google.com/file/d/1sılouxjza6rgv_ xsAlfzdsm_f4_psebh/view? usp=sharing, con el objetivo de implementar el modelo didáctico alternativo y la corriente pedagógica cognitivista, ya que durante la contextualización se logró inferir que estas eran las que más podían ajustarse a las necesidades del contexto

En la figura 1 se muestran algunas imágenes referentes a la estructura de la unidad didáctica.

Figura 1. Partes de la estructura de la unidad didáctica. (Fotografía de A. Vargas, 2020).
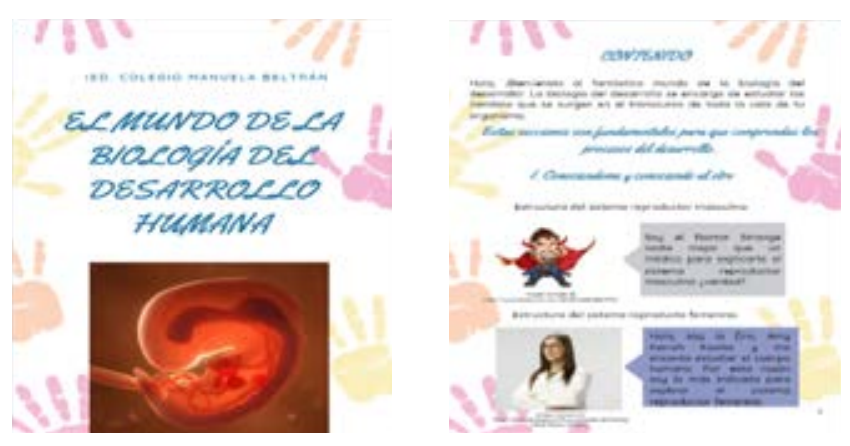

Los personajes de ficción fueron elegidos porque están relacionados con la ciencia y puedan hacer que los consejos y algunos conceptos fueran más dinámicos. Debido a la situación que se presenta por la covid-19, la unidad didáctica se diseñó considerando lo analizado en la contextualización, pues la institución no permitió el acceso de practicantes a sus plataformas virtuales, lo que impidió su implementación. Cabe resaltar que se abordan algunos conceptos en ciertos casos de forma aislada y general, puesto que este material didáctico requiere la asesoría de algún docente que esté atento a las dudas o demás aspectos que surjan en el aula.

También se destaca que para lograr los objetivos propuestos en la unidad didáctica se requiere un periodo aproximado de dos meses, puesto que el material incluye guías, datos, documentales y fragmentos de series. El tiempo podría ser un factor limitante a la hora de su ejecución.

La estructura de la unidad didáctica ofrece al maestro una organización y planeación de actividades que permitan ver el progreso que el estudiante tiene frente a los conceptos que conforman la biología del desarrollo humano, por lo cual se evidencia un modelo pedagógico cognitivista.

\section{Conclusiones}

- El modelo didáctico alternativo y la corriente pedagógica cognitiva permitieron realizar una didáctica en la que se destaca la importancia de abordar los
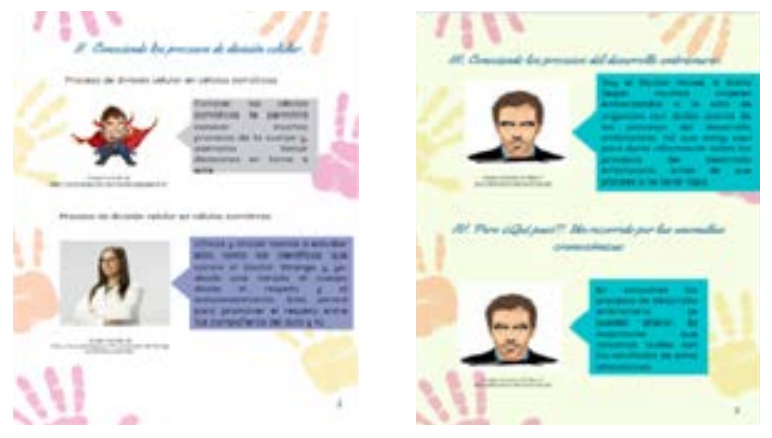

conceptos de determinado tema. Además, ofrece la posibilidad de dar consejos o recomendaciones para el cuidado del cuerpo humano y formular situaciones hipotéticas que se pueden presentar en el contexto de los estudiantes.

- La indagación de las ideas previas a través de material didáctico ayuda a comprender la forma en que los estudiantes se acercan al conocimiento desde la cotidianidad, pues no se enfoca en preguntar conceptos, sino en ver cómo pueden resolver o analizar determinado problema o concepto.

- Se realizó un acercamiento desde las realidades contextuales de los estudiantes de octavo grado del Colegio Manuela Beltrán relacionadas con la biología del desarrollo - cuidado personal, sexualidad, alimentación sana y casos de embarazo a temprana edad (que no se presentaron en el aula, pero sí en la institución)-, de ahí surgió la necesidad de abordar temas que se presentaron.

- La biología del desarrollo es una temática con potencial para contribuir al conocimiento del cuerpo humano en la población estudiantil de octavo grado del Colegio Manuela Beltrán, pues permite que lo aprendido les ayude a tomar decisiones frente al cuidado de su cuerpo en aspectos como la alimentación, el consumo de sustancias psicoactivas y la sexualidad.

- Si bien esta unidad didáctica es una alternativa para un contexto en el que los estudiantes usan ampliamente las TIC, esto requiere del conocimiento de 
plataformas que permitan un formato de evaluación más interactivo y dinámico para los estudiantes. De esta manera, el docente podrá usar la tecnología a su favory no en su contra (como distractor en clase por el uso de redes sociales).

- Las guías elaboradas permiten conocer las ideas previas de los estudiantes y ayudan a conocer los posibles errores conceptuales que se pueden presentar en un tema tan amplio y complejo como el de la biología del desarrollo humano.

\section{Referencias}

Cáceres Péfaur, B., Del Valle Carballo Pérez, K. y Péfaur Vega, J. E. (2016). La sistematización de la unidad didáctica en educación ambiental: una aproximación desde una experiencia en la ruralidad. Educere, 20(66), 249-257.

Peláez Cárdenas, D. (2017). Mi experiencia como maestra en formación en la construcción y aplicación de una unidad didáctica. Bio-grafía, 10(19), 257-264. https://doi.org/10.17227/bio-grafia. extra2017-7114

Cerda, H. (1993). Los elementos de la investigación. Cómo reconocerlos, diseñarlos y construirlos. Editorial el Búho.

Colegio Manuela Beltrán. (2018). Proyecto Educativo Institucional. https://www.redacademica.edu.co/ colegios/colegio-manuela-beltran-ied.

Curtis, H. (2015). Biología. Editorial Médica Panamericana.
Gómez Hurtado, M. y Polanía González, N. R. (2008). Estilos de enseñanza y modelos pedagógicos. Un estudio con profesores del Programa de Ingeniería Financiera de la Universidad Piloto de Colombia. [Tesis de Maestría, Universidad de la Salle]. Repositorio institucional Unisalle https://ciencia. lasalle.edu.co/maest_docencia/554/

Mayorga Fernández, M. J. y Madrid Vivar, D. (2015). Modelos didácticos y Estrategias de enseñanza en el Espacio Europeo de Educación Superior. Tendencias Pedagógicas, 15, 91-111. https://revistas.uam. es/tendenciaspedagogicas/article/view/1934

Ministerio de Educación Nacional. (2016). Derechos Básicos de Aprendizaje-Ciencias Naturales. http:// aprende.colombiaaprende.edu.co/sites/default/ files/naspublic/DBA_C.Naturales.pdf.

Ríos, G. V. y Urdaneta, H. C. (2015). Actual vigencia de los modelos pedagógicos en el contexto educativo. Opción: Revista de Ciencias Humanas y Sociales, 31(6), 914-934.

Rodríguez, M., Fernández, M. T. y Rivadeneira, F. (s. f.). Agentes teratogénicos y teratogenicidad, (10). Colección Derecho a Vivir en Desventaja.

Sadler, T. W. y Langman, J. (2007). Langman: Embriología médica con orientación clínica. Editorial Médica Panamericana.

Sánchez, F., (2015). Adolescencia. Necesidades y problemas. Implicaciones para la intervención. Adolescere, 3(2), 09-17.

Tortora, G. J. y Derrickson, B. (2013). Principios de anatomía y fisiología. Editorial Médica Panamericana. 